\title{
Cost Effectiveness of Human Papillomavirus-16/18 Genotyping in Cervical Cancer Screening
}

\author{
Warner K. Huh $\cdot$ Erin Williams $\cdot$ Joice Huang • \\ Tommy Bramley $\cdot$ Nick Poulios
}

Published online: 11 November 2014

(C) Springer International Publishing Switzerland 2014

\begin{abstract}
Background There is limited understanding of the health economic implications of cervical screening with human papillomavirus (HPV)-16/18 genotyping.

Objective The aim of this study was to evaluate the cost effectiveness of cervical cancer primary screening with a HPV-16/18 genotyping test which simultaneously detects 12 other high-risk HPV types.

Methods A Markov cohort model compared four strategies: (1) cytology with reflex HPV testing for atypical squamous cells of undetermined significance (ASC-US); (2) co-testing with cytology and HPV testing; (3) HPV with reflex to cytology; and (4) HPV with 16/18 genotyping and reflex cytology (ASC-US threshold). Screening began at age 30 and was performed triennially over 40 years. Screening sensitivity and specificity values for cervical intraepithelial neoplasia (CIN) 3 were obtained from the Addressing THE Need for Advanced HPV Diagnostics (ATHENA) trial. Outcomes for a 1-year follow-up scenario wherein persistent disease was detected were estimated. Screening and cancer treatment costs were calculated from
\end{abstract}

\footnotetext{
W. K. Huh $(\bowtie)$

University of Alabama at Birmingham, 1700 6th Avenue South, WIC Room 10250, Birmingham 35233, AL, USA

e-mail:whuh@uabmc.edu

E. Williams · T. Bramley

Xcenda, 4114 Woodlands Parkway, Palm Harbor,

FL 34685, USA

J. Huang · N. Poulios

Roche Molecular Diagnostics, Pleasanton, CA, USA
}

a US payer's perspective in 2013. Costs and qualityadjusted life-years (QALYs) were discounted at $3 \%$ annually.

Results Applying a US\$50,000/QALY threshold, strategy (4) dominated strategies (2) and (3) by reducing costs and cancer incidence and improving QALYs, and was cost effective versus strategy (1). Accounting for persistent $\geq$ CIN 3 at 1 year, strategy (4) was cost effective versus all other strategies. Detecting HPV-16/18 resulted in earlier diagnosis of clinically relevant $\geq$ CIN 3 at initial screening and efficient use of follow-up resources. Outcomes were most influenced by strategy performance.

Conclusions Incorporating HPV-16/18 genotyping is cost effective and may improve detection of CIN, thereby preventing cervical cancer.

\section{Key Points for Decision Makers}

Access to cervical cancer screening strategies that facilitate early detection of clinically relevant cervical intraepithelial neoplasia associated with HPV-16/18 allows women to seek treatment sooner and may thereby improve cancer protection.

Stratifying cancer risk via HPV-16/18 genotyping in women aged $\geq 30$ years may be cost saving compared with cotesting with cytology and HPV testing by reducing the number of screening tests and overall screening costs.

HPV-16/18 genotyping with reflex cytology is cost effective in cervical screening and represents a beneficial alternative approach to cervical screening from both a health and economic perspective. 


\section{Introduction}

Organized screening programs aimed at early detection of high-grade cervical intraepithelial neoplasia (CIN) have steadily reduced cervical cancer mortality (Alliance for Cervical Cancer Prevention, Preventing Cervical Cancer Worldwide, 2004). Nonetheless, individual screening tests can suffer from shortcomings, particularly when applied indiscriminately to the general screening population. Subjective interpretation of Pap cytology ('cytology') renders a high degree of variability [1]. In populations with low prevalence of human papillomavirus (HPV), cytology demonstrates low sensitivity and poorer positive predictive value due to fewer high-grade lesions [1]. Also, primary HPV pooled testing exhibits lower specificity in excluding the absence of high-grade CIN [2]. Accordingly, ongoing efforts aim to identify strategies incorporating more prudent use of individual screening tests.

Since HPV-16 and HPV-18 account for more than $70 \%$ of cervical cancer cases [3, 4] and confer a higher risk for neoplasia [5], testing for these genotypes may be clinically useful for stratifying cancer risk [6]. A qualitative in vitro assay (cobas ${ }^{\circledR}$ HPV Test), which simultaneously detects 14 high-risk HPV types and provides specific genotyping information for HPV-16 and HPV-18, was validated in the Addressing THE Need for Advanced HPV Diagnostics (ATHENA) trial $[6,7]$. The trial concluded that primary HPV testing and triage of HPV-positive women with HPV16/18 genotyping and cytology may balance screening benefits and harms by maximizing sensitivity while limiting the number of diagnostic tests [7]. The US Food and Drug Administration recently approved the cobas ${ }^{\circledR} \mathrm{HPV}$ Test for primary cervical cancer screening in women aged $\geq 25$ years. To explore the economic implications, we compared the relative cost effectiveness of HPV-16/18 genotyping and three other primary cervical screening strategies.

\section{Materials and Methods}

\subsection{Study Population}

The model evaluated the outcomes of a hypothetical cohort of 1,000 non-hysterectomized women who were asymptomatic for cervical cancer and had participated in cervical screening in a US healthcare setting over a 40 -year period. The starting age of the cohort was 30 years. Because the risk of HPV persistence increases with age [8], HPV testing in women aged $\geq 30$ years may identify more clinically significant infections and, therefore, the performance of HPV$16 / 18$ genotyping in this population is of particular interest.

\subsection{Comparator Screening Strategies}

We assessed the cost effectiveness of four primary cervical screening strategies: (1) cytology with reflex HPV testing for triage of atypical squamous cells of undetermined significance (ASC-US) ('Cytology'); (2) co-testing with cytology and HPV testing ('Co-testing'); (3) HPV with reflex cytology for positive HPV ('HPV HR [human papillomavirus, high-risk] Only'); and (4) HPV with 16/18 genotyping and reflex cytology (ASC-US threshold) ('HPV with Genotyping'). (See Fig. 1 for detailed algorithms). Cytology and Co-testing were selected based on the American Cancer Society (ACS)-American Society for Colposcopy and Cervical Pathology (ASCCP)-American Society of Clinical Pathologists (ASCP) guidelines. These strategies represented practices most likely to be implemented in community settings $[9,10]$.

Routine screening was performed triennially with some women returning for 1-year retesting based on primary screening results. We assumed that all women complied with scheduled screening visits as well as recommendations for colposcopy and biopsy. For all strategies, women with false positive results returned for follow-up screening as recommended by the ASCCP [9]. Women exited screening at age 70 years in the model based on clinical guidelines advising cessation of screening after the age of 65 years, but extending beyond age 65 for women with a history of CIN 2, CIN 3, or cervical cancer [9].

\subsection{Markov Model Structure and Health States}

The Excel-based Markov cohort model (Microsoft Corporation, Redmond, Washington) incorporated a 'no-screening' model which simulated the natural history of oncogenic HPV infection in an unscreened population. This natural history model served as the framework within which the effects of each screening strategy were applied and the outcomes compared. Since the analysis reflected clinical practice in the US, 'no screening' was not considered a comparator option.

Applying literature-based transition probabilities [1119], women transitioned annually across seven possible states: (1) No high-risk HPV infection; (2) High-risk HPV infection (no CIN); (3) CIN 1; (4) CIN 2; (5) CIN 3 or worse; (6) Cervical Cancer, and (7) Dead (Fig. 2). At baseline, the cohort was apportioned among the Markov states based on the age-adjusted prevalence of high-risk HPV infection, the probability of CIN, and the age-adjusted cervical cancer incidence (Table 1). Age-specific probabilities for high-risk HPV infection and CIN clearance, progression, and regression were obtained from published epidemiologic, cost-effectiveness, and mathematical models simulating HPV infection and cervical 
Fig. 1 Cost-effectiveness analysis of cervical screening strategies in non-

hysterectomized women aged $\geq 30$ years: primary screening algorithms. a Cytology with reflex HPV at ASC-US threshold ('Cytology') (Women who had ASC-US during primary screening with Cytology were triaged to HPV testing, whereas those with cytology findings worse than ASC-US were referred to colposcopy). b Co-screening with reflex for ASC-US ('Cotesting') (In Co-testing, women were referred to colposcopy if they had either (i) ASC-US and a positive HPV test, or (ii) cytology worse than ASC-US regardless of HPV status). c HPV with reflex to cytology at ASC-US threshold ('HPV HR Only') (In HPV HR Only, all women who tested positive for HPV were further evaluated with cytology. Those with normal cytology were retested in 1 year, whereas women exhibiting ASC-US or worse were referred to colposcopy). d HPV-16/18 genotyping and reflex cytology at ASC-US threshold ('HPV with Genotyping') (Women with HPV-16 or HPV-18 detected by HPV with Genotyping during primary screening were referred for immediate colposcopy, whereas those testing positive for other high-risk HPV genotypes but with normal cytology were retested at 1 year). ASC-US atypical squamous cells of undetermined significance, $H P V$ human papillomavirus, $H P V H R$ human papillomavirus, high-risk, NILM negative for intraepithelial lesion or malignancy (a)

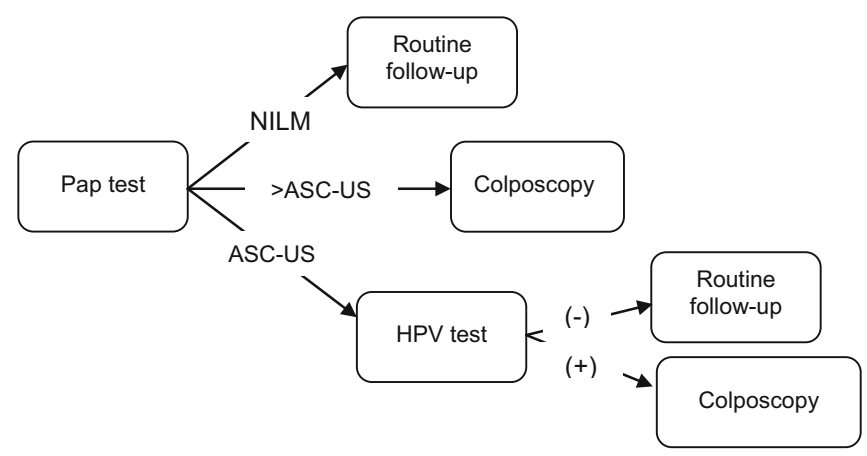

(b)

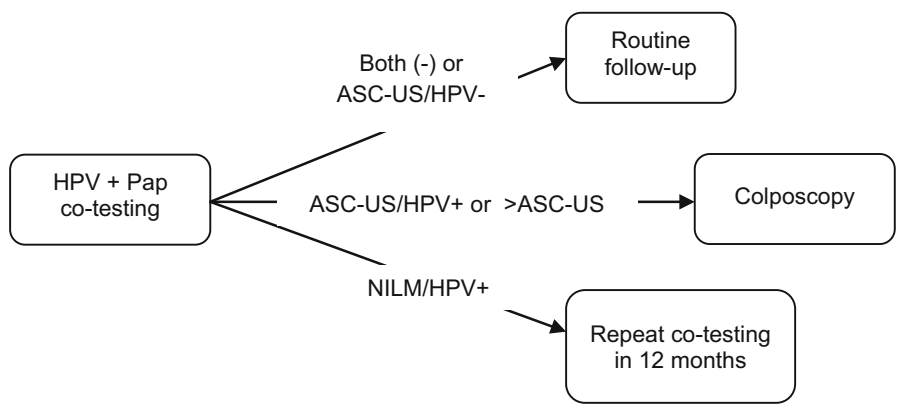

(c)

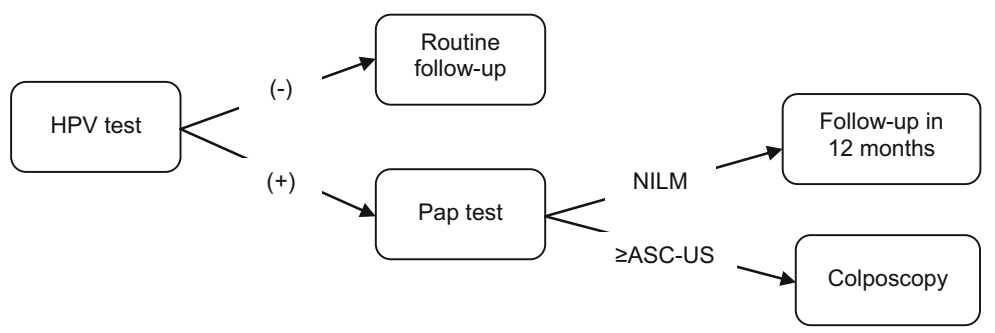

(d)

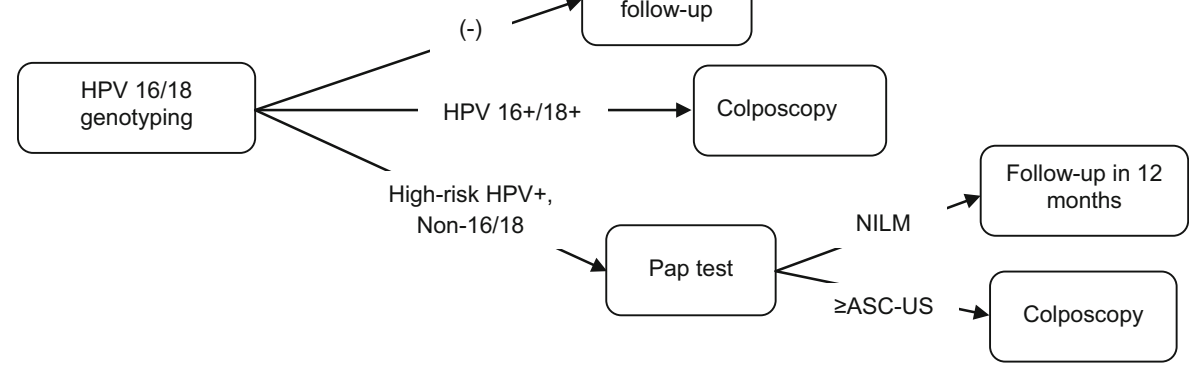

carcinogenesis (Table 1) [17-23]. Annual transition probabilities for progression to CIN 2 and $\geq$ CIN 3 from no CIN were computed from the 36-month cumulative hazard rate for progression from incident HPV-16 infection to CIN 2 and $\geq$ CIN $3(16.5 \%)$ reported for the placebo arm $(n=42)$ in a randomized controlled trial of a HPV-16 vaccine [21]. Progression to invasive cervical cancer occurred within a single cycle from $\geq \mathrm{CIN} 3$ only, whereas 

cervical intraepithelial neoplasia, $H P V$ human papillomavirus
Fig. 2 Markov health states for oncogenic HPV infection. CIN

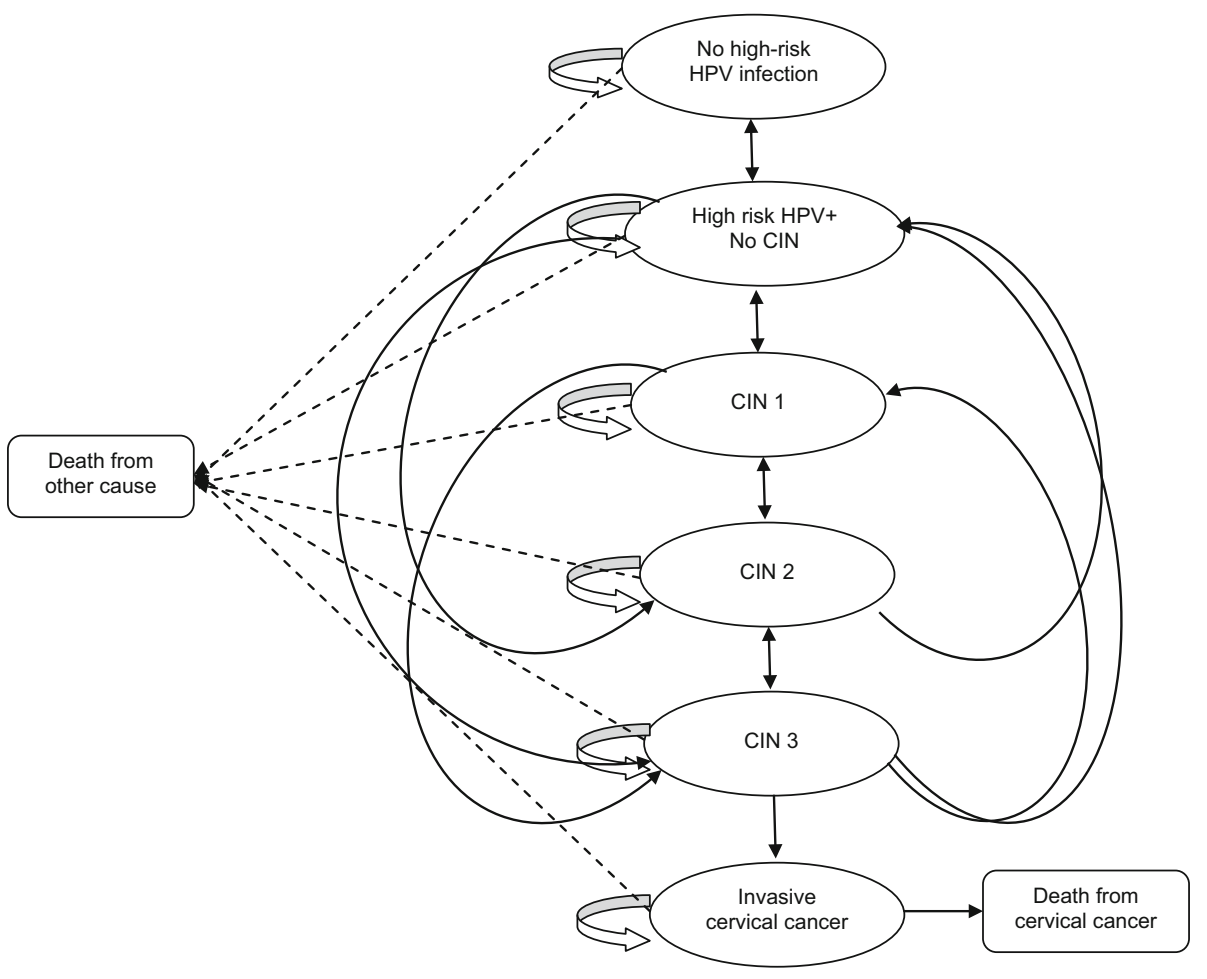

regression from Cervical Cancer to the CIN states did not occur. Given that we primarily intended to address the screening effect of HPV with Genotyping, we did not stratify Cervical Cancer by stage; rather, we modeled health states for pre-cancer lesions arising from persistent HPV infection. Other models utilizing a single health state to depict invasive cervical cancer have also been published in the literature [24, 25].

The no-screening model was manually calibrated to approximate published estimates of cervical cancer incidence and mortality in an unscreened population. In calibrating a natural history model for HPV infection and cervical cancer, Taylor et al. [26] noted that manual calibration was one of two methods achieving the best fit, yielding a $10 \%$ mean deviation from optimal fit. Our final model generated an annual cervical cancer incidence of approximately 19.47 cases per 100,000 women-years. This projection was $10.1 \%$ higher than the age-standardized incidence rate of 17.70 per 100,0000 women-years for an unscreened US population cited by Gustafsson et al. [27], but $1.1 \%$ lower than the incidence (20.64 per 100,000 ) predicted by a cost-effectiveness screening model by Vijayaraghavan et al. [11].

Age-adjusted annual probabilities of death for women without cervical cancer were derived from the general population estimates reported in the US Social Security Actuarial Publications Period Life Table. To estimate cervical cancer mortality in the unscreened population, the age-specific 5-year relative cervical cancer survival rates reported by the National Cancer Institute Surveillance
Epidemiology and End Results (SEER) Cancer Statistics Review (1975-2007) were converted to annual probabilities and multiplied by the respective annual numbers of expected cervical cancer cases [28]. Our model estimated an annual cervical cancer mortality rate of 15.5 per 100,000 unscreened women. Published cervical cancer mortality rates in unscreened populations vary by geographic locality, population age, and study year, but have exceeded 20 deaths per 100,000 women in some studies [29]. For the screening cohorts, women who were diagnosed with cervical cancer were assigned a 5-year relative survival rate of $90 \%$ per the ACS based on the assumption that cancers in asymptomatic women receiving regular screening with $100 \%$ compliance would tend to be early-stage, curable cancers (ACS, Cancer Facts and Figures, 2010) [30].

In addition to face validation of the no-screening model, analytical methods (including programming, coding, and equations), configuration of screening algorithms, screening results, and costs were reviewed by individuals with expertise in the area of cervical cancer screening (including two clinicians) and were found to be reasonable within the structural limitations of a Markov cohort model.

\subsection{Model Inputs}

\subsubsection{Screening Performance and Key Assumptions}

Baseline performance values were obtained from the ATHENA trial, the largest US-based screening accuracy 
Table 1 Model assumptions for the prevalence of high-risk HPV, HPV-16/18, and health state transition probabilities by age group

\begin{tabular}{|c|c|c|c|c|c|c|c|c|c|c|}
\hline & \multicolumn{9}{|c|}{ Age group (years) } & \multirow[t]{2}{*}{ Reference(s) } \\
\hline & $25-29$ & $30-34$ & $35-39$ & $40-44$ & $45-49$ & $50-54$ & $55-59$ & $60-64$ & $65-70$ & \\
\hline \multicolumn{11}{|l|}{ Prevalence } \\
\hline HPV HR, \% infected & 21.1 & 13.4 & 9.9 & 7.6 & 6.6 & 6.6 & 5.9 & 5.9 & 4.4 & ATHENA [12] \\
\hline HPV-16/18, $\%$ of HPV HR & 33.0 & 28.6 & 27.9 & 20.3 & 23.8 & 19.9 & 19.3 & 18.1 & 18.9 & ATHENA [12] \\
\hline \multicolumn{11}{|l|}{ HPV HR, \% per cytology } \\
\hline Normal & 16.6 & 10.6 & 7.5 & 6.1 & 5.4 & 5.6 & 5.0 & 5.3 & 4.0 & ATHENA [12] \\
\hline ASC-US & 49.4 & 31.2 & 28.5 & 19.8 & 9.8 & 18.8 & 20.3 & 20.0 & 12.5 & ATHENA [12] \\
\hline LSIL/HSIL & 78.7 & 72.6 & 69.0 & 61.5 & 60.6 & 56.1 & 65.5 & 50.0 & 66.7 & ATHENA [12] \\
\hline \multicolumn{11}{|c|}{ Annual transition probabilities used in the model } \\
\hline HPV HR (-) to HPV HR (+) & 0.1500 & 0.0576 & 0.0333 & 0.0333 & 0.0333 & 0.0222 & 0.0222 & 0.0222 & 0.0222 & {$[17,20,23]$} \\
\hline HPV HR (+) to HPV HR (-) & 0.7000 & 0.4130 & 0.4130 & 0.4130 & 0.4130 & 0.4130 & 0.4130 & 0.4130 & 0.4130 & {$[17,20-22]$} \\
\hline HPV HR (+) no CIN to CIN1 & 0.1100 & 0.1100 & 0.1100 & 0.1100 & 0.1100 & 0.1100 & 0.1100 & 0.1100 & 0.1100 & {$[18,20]$} \\
\hline $\begin{array}{l}\text { HPV HR (+) no CIN to CIN } \\
2 \text { or CIN } 3\end{array}$ & 0.0583 & 0.0583 & 0.0583 & 0.0583 & 0.0583 & 0.0583 & 0.0583 & 0.0583 & 0.0583 & {$[18,21]$} \\
\hline CIN 1 to CIN 2 or CIN 3 & 0.0198 & 0.0198 & 0.1444 & 0.1444 & 0.2688 & 0.2688 & 0.2688 & 0.2688 & 0.2688 & {$[19,21]$} \\
\hline $\begin{array}{l}\text { CIN } 2 \text { or CIN } 3 \text { to Cervical } \\
\text { Cancer }\end{array}$ & 0.0060 & 0.0060 & 0.0060 & 0.0060 & 0.0060 & 0.0060 & 0.0060 & 0.0060 & 0.0060 & {$[18,20,21]$} \\
\hline $\begin{array}{l}\text { CIN1 to HPV HR }(+) \text { or HPV } \\
\text { HR }(-)\end{array}$ & 0.2248 & 0.2248 & 0.1124 & 0.1124 & 0.1124 & 0.1124 & 0.1124 & 0.1124 & 0.1124 & [17] \\
\hline CIN 2 or CIN 3 to CIN1 & 0.2340 & 0.2340 & 0.2340 & 0.2340 & 0.2340 & 0.2340 & 0.2340 & 0.2340 & 0.2340 & {$[17,20]$} \\
\hline $\begin{array}{l}\text { CIN } 2 \text { or CIN } 3 \text { to HPV } \\
\text { HR }(-)\end{array}$ & 0.2036 & 0.2036 & 0.2036 & 0.2036 & 0.1901 & 0.1901 & 0.1901 & 0.1901 & 0.1901 & {$[17]$} \\
\hline
\end{tabular}

ASC-US atypical squamous cells of undetermined significance, ATHENA Addressing THE Need for Advanced HPV Diagnostics, CIN cervical intraepithelial neoplasia, $H P V$ human papillomavirus, $H R$ high risk, $H S I L$ high-grade squamous intraepithelial lesion, $L S I L$ low-grade squamous intraepithelial lesion

cohort study for HPV-16/18 genotyping which included more than 47,000 women ages $\geq 21$ years from clinical sites across 23 states [7, 12]. A subanalysis of 34,254 participants aged $\geq 30$ years (mean 44.7 years) compared ten different cervical screening strategies for detecting high-grade cervical disease [7]. In addition to HPV-16/18 genotyping, the analyses evaluated strategies without HPV$16 / 18$ genotyping that were considered of interest in clinical practice (several of which were included in the present model). Given the large cohort, expected frequency of CIN [12], and pertinent strategies evaluated in a single population, the ATHENA trial was considered an appropriate source for screening performance data.

Screening performance was defined as the sensitivity and specificity for $\geq$ CIN 3 and referred to overall strategy performance as opposed to that of component tests. Since approximately $90 \%$ of CIN 1 and $88 \%$ of CIN 2 cases regress within 1 year $[31,32]$, the model accounted only for sensitivity in detecting incident and persistent cases of $\geq$ CIN 3. At publication, the ATHENA analysis reported only the baseline performance of the strategies as representing usual clinical practice. Thus, comparing the 'true' sensitivities (defined as performance achieved by completing a full screening cycle) was not feasible since outcomes could be only partially ascertained from the initial screening. Therefore, we examined a scenario wherein all cases deferred at baseline returned for 1-year follow-up. Screening sensitivities were re-calculated by assuming that deferred $\geq$ CIN 3 cases would be captured at the 1 -year visit. Detecting additional $\geq$ CIN 3 effectively increased the overall sensitivities of the strategies, particularly those deferring more patients at baseline; this resulted in similar performance between the HPV with Genotyping and HPV HR Only strategies and improved sensitivity for co-testing (Table 2).

\subsubsection{Costs}

Costs were modeled from the perspective of the US healthcare payer and reported in 2013 US dollars (Table 2). Unit costs represented the median national Medicare payments for the respective Common Procedural Terminology codes (Centers for Medicare and Medicaid Services Physician Fee Schedule, 2013). For each strategy, the total annual cost included the costs for screening (including routine examinations, triage, and retesting) and treatment for CIN and cervical cancer. Screening costs included the costs for individual tests and office visits. Screening and 
treatment costs were calculated by multiplying the relevant unit costs by the respective expected percentages of women utilizing each test or treatment in the strategy, and then summing the utilization-weighted costs.

\subsubsection{Health Utilities}

Quality-adjusted life-years (QALYs) were calculated by multiplying the expected number of life-years gained by the assigned health utility for each Markov state (Table 2). Health utility values for the CIN 1 and the CIN 2 and CIN 3 states represented the utilities for low-grade squamous intraepithelial lesions and high-grade squamous intraepithelial lesions, respectively, as cited by Sanders et al. [18] and applied to the US population in a study by the Institute of Medicine (IOM) [33]. In the latter study, Health Utilities Mark II utilities, which were derived for a general Canadian population via the standard gamble method, were assigned quality-adjusted weights to valuate various morbidity states in the US [33]. To avoid overestimating the utility for Cervical Cancer, we assigned a base value of 0.71 which represented the mean weighted health utility for cervical cancer in the treatment phase $(0.79$ for Stage I and 0.62 for Stages II-IV per Sanders et al.) adjusted by the SEER stage distribution for cervical cancer at diagnosis (see Table 2) [19, 28]. We assumed a 1-year disutility for detected but asymptomatic cervical cancer cases [19].

\subsection{Model Outcomes}

Outcomes included direct medical costs and QALYs calculated over 40 years and discounted at a yearly rate of $3.0 \%[34,35]$. Cost effectiveness was expressed as the incremental cost-effectiveness ratio (ICER), which represented the additional cost per QALY gained using one strategy versus the next non-dominated strategy, and assessed against the payer's willingness-to-pay (WTP) criterion of US $\$ 50,000 /$ QALY. Other outcomes included the undiscounted number of life-years gained, annual cervical cancer incidence, and mortality rate (per 100,000 women) for each strategy. Additionally, we determined the expected number of colposcopies required to detect one case of $\geq \mathrm{CIN} 3$, an endpoint considered by the ACS-ASCCP-ASCP guidelines as a primary surrogate for the potential harm of cervical screening [9].

\subsection{Sensitivity Analyses}

We employed one-way sensitivity analyses to assess the impact of key inputs on the relative cost effectiveness of HPV with Genotyping versus the comparators. The resulting tornado diagrams depicted the degree of influence of each parameter on the relative cost effectiveness of HPV with Genotyping when tested at high $(+10 \%)$ and low $(-10 \%)$ values; wider bars indicated greater influence. Additionally, we performed threshold analyses using an Excel what-if analysis function to examine the effects of further increasing or decreasing parameters exhibiting greater influence on visual inspection or those of particular interest (such as sensitivity and costs).

To quantify the likelihood that HPV with Genotyping is cost effective compared with the non-dominated strategies, probabilistic sensitivity analysis (PSA) was performed by simultaneously varying key inputs across values sampled 1,000 times from assigned distributions (Table 2). We applied a standard error (SE) of $1.0 \%$ for sensitivities and specificities (based on the ATHENA data $[n=34,254]$ ), and in the absence of other data, assumed a SE of $10 \%$ the deterministic value for costs, health utilities, and the annual discount rate for costs and effects. The resulting costeffectiveness acceptability curve showed the percentage likelihood that the strategy was cost effective when assessed against WTP threshold criteria. We used the net monetary benefit (NMB), a measure deemed appropriate by the International Society for Pharmacoeconomics and Outcomes Research, for generating the results of the PSA [36]. When comparing two strategies, the strategy generating a greater NMB (positive incremental NMB [INMB]) was considered more cost effective.

\section{Results}

In the primary analysis, HPV with Genotyping was more effective and less costly compared with both Co-testing and HPV HR Only, thereby dominating the two strategies (Table 3). Compared with Cytology, HPV with Genotyping was cost effective, generating an acceptable ICER of US\$7,667/QALY. Further, HPV with Genotyping appeared beneficial in reducing annual cervical cancer incidence and mortality rates compared with the other strategies, while requiring a relatively moderate number of colposcopies for each $\geq$ CIN 3 detected (Table 3 ).

As shown in Table 4, women were more frequently triaged with Cytology when HPV HR Only was employed for primary screening compared with HPV with Genotyping. Similarly, fewer women screened with HPV with Genotyping were deferred to 1-year retesting compared with those triaged in both HPV HR Only and Co-testing. Examining the expenditures (data not shown), the average total costs which included routine and repeat screening, triage tests (for HPV HR Only), colposcopy, and biopsy, were higher for Co-testing and HPV HR Only (US\$1,737 and US $\$ 1,481$, respectively) compared with Cytology and HPV with Genotyping (US\$1,001 and US\$1,064, respectively). 
Table 2 Key model inputs and assumptions

\begin{tabular}{|c|c|c|c|c|c|}
\hline Variable & Base & Range & Distribution & \multicolumn{2}{|l|}{ Reference(s) } \\
\hline \\
\hline Effects and costs & $3.0 \%$ & $2.7-5.0 \%$ & Normal & \multicolumn{2}{|l|}{ Assumption } \\
\hline \multicolumn{6}{|l|}{ Unit costs (2013 USD) } \\
\hline Office visit (routine/repeat screening) & $\$ 72.81$ & $\$ 65.53-\$ 80.09$ & Normal & \multicolumn{2}{|c|}{ CPT 99213 (office visit, established patient) } \\
\hline Cytology & $\$ 27.85$ & $\$ 25.07-\$ 30.64$ & Normal & \multirow{2}{*}{\multicolumn{2}{|c|}{$\begin{array}{l}\text { CPT } 88142,88143 \text { (cytopathology, cervical or vaginal) } \\
\text { CPT } 87621 \text { (agent detection by nucleic acid; } \\
\text { papillomavirus, human, amplified probe technique) }\end{array}$}} \\
\hline HPV DNA pooled test & $\$ 48.24$ & $\$ 43.42-\$ 53.06$ & Normal & & \\
\hline HPV-16/18 genotyping test & $\$ 48.24$ & $\$ 43.42-\$ 53.06$ & Normal & \multicolumn{2}{|l|}{ Same as above } \\
\hline Colposcopy plus biopsy & $\$ 287.67^{\mathrm{a}}$ & $\$ 258.90-\$ 316.44$ & Normal & \multicolumn{2}{|c|}{ CPT 57455 (colposcopy and biopsy of cervix) } \\
\hline Treatment for $\geq \mathrm{CIN} 3$ & $\$ 1,292^{\mathrm{b}}$ & $\$ 1,162-\$ 1,421.20$ & Normal & \multicolumn{2}{|l|}{$[11]$} \\
\hline Cervical cancer treatment & $\$ 47,840^{\mathrm{b}}$ & $\$ 40,445-\$ 49,432.90$ & Normal & \multicolumn{2}{|l|}{$[11,16]$} \\
\hline \multicolumn{6}{|l|}{ Sensitivity for $\geq$ CIN 3} \\
\hline Cytology (with reflex HPV test [ASC-US]) & $56.1 \%$ & $50.5-61.7 \%$ & Beta & ATHENA [7] & \\
\hline $\begin{array}{l}\text { HPV with genotyping (reflex cytology } \\
\text { [ASC-US]) }\end{array}$ & $72.0 \%$ & $64.8-79.2 \%$ & Beta & ATHENA [7] & \\
\hline HPV HR only (with reflex to cytology) & $51.9 \%$ & $46.7-57.1 \%$ & Beta & ATHENA [7] & \\
\hline Co-testing (with reflex for ASC-US) & $56.1 \%$ & $50.5-61.7 \%$ & Beta & ATHENA [7] & \\
\hline Specificity for $\geq$ CIN 3 & & & & & \\
\hline Cytology (with reflex HPV test [ASC-US]), & $87.6 \%$ & $78.8-96.4 \%$ & Beta & ATHENA [7] & \\
\hline $\begin{array}{l}\text { HPV with genotyping (reflex cytology } \\
\text { [ASC-US]) }\end{array}$ & $85.2 \%$ & $76.7-93.7 \%$ & Beta & ATHENA [7] & \\
\hline HPV HR only (with reflex to cytology) & $91.3 \%$ & $82.2-100 \%$ & Beta & ATHENA [7] & \\
\hline Co-testing (with reflex for ASC-US) & $87.6 \%$ & $78.8-96.4 \%$ & Beta & ATHENA [7] & \\
\hline Estimated sensitivities for 1 -year follow-up sce & nario & Base $(\%)$ & Range & Distribution & Formula for calculation $^{\mathrm{c}}$ \\
\hline Cytology (with reflex HPV test [ASC-US]) ${ }^{\mathrm{d}}$ & & 56.1 & & & $(106+0) / 189=56.1 \%$ \\
\hline HPV with genotyping (reflex cytology [ASC-U & & 89.9 & & & $(136+34) / 189=89.9 \%$ \\
\hline HPV HR only (with reflex to cytology) & & 89.9 & & & $(98+72) / 189=89.9 \%$ \\
\hline Co-testing (with reflex for ASC-US) & & 94.2 & & & $(106+72) / 189=94.2 \%$ \\
\hline Health utilities & & Range & & Distribution & Reference(s) \\
\hline Well & & $0.90-1.0$ & & Beta & [19] \\
\hline CIN 1 & & $0.87-1.0$ & & Beta & [19] \\
\hline CIN 2 & & $0.87-1.0$ & & Beta & [19] \\
\hline CIN 3 & & $0.87-1.0$ & & Beta & [19] \\
\hline Cervical cancer & & $0.64-0.78$ & & Beta & {$[19,28]$} \\
\hline
\end{tabular}

ASC-US atypical squamous cells of undetermined significance, ATHENA Addressing THE Need for Advanced HPV Diagnostics, CIN cervical intraepithelial neoplasia, $C P T$ common procedural terminology, DNA deoxyribonucleic acid, HPV human papillomavirus, HPV HR human papillomavirus, high-risk, SEER National Cancer Institute Surveillance Epidemiology and End Results, USD United States dollars

${ }^{a}$ Estimated cost includes physician's fee (CPT 88305, US\$70.09). Model assumed ratio of colposcopies to biopsies $=1.5 ; 82.6 \%$ of colposcopy/biopsy procedures were performed in physicians' offices and $17.4 \%$ were performed in hospital outpatient settings

${ }^{\mathrm{b}}$ Costs were adjusted to 2013 USD using the medical component of the Consumer Pricing Index

${ }^{c}$ Adjusted sensitivity values were calculated as (baseline +1 -year follow-up)/total $\geq$ CIN 3, where baseline and 1-year follow-up signified the number of $\geq$ CIN 3 cases detected at baseline screening and 1-year follow-up, respectively, and total $\geq$ CIN 3 (denominator) indicated the total cumulative number of $\geq$ CIN 3 cases prevalent in the ATHENA trial cohort as confirmed by colposcopy and valid biopsy

${ }^{\mathrm{d}}$ This strategy does not include retesting at 1 year. Women with >ASC-US or who have ASC-US and are high-risk HPV-positive are referred for immediate colposcopy

e Assumption was based on health utilities for cervical cancer in treatment phase ( 0.79 for Stage I and 0.62 for Stages II-IV), weighted by SEER stage distribution for cervical cancer at diagnosis; value represents weighted mean health utility. Assumed $5 \%$ unstaged patients were distributed as follows: $2 \%$ local; $2 \%$ regional; and $2 \%$ distant disease

Similar trends were observed in the second scenario. Despite equivalent sensitivities, HPV with Genotyping resulted in fewer women being triaged and deferred to follow-up compared with HPV HR Only, yielding lower average screening costs (US $\$ 1,063$ versus US $\$ 1,463$, respectively) and an average cost-savings of US $\$ 400$ per 
Table 3 Cost effectiveness and outcomes of four cervical screening strategies in women aged $\geq 30$ years

\begin{tabular}{|c|c|c|c|c|c|c|}
\hline Screening strategy & $\begin{array}{l}\text { Discounted } \\
\text { Cost }^{\mathrm{a}}\end{array}$ & QALYs & $\begin{array}{l}\text { ICER } \\
(\$ / Q A L Y)^{a}\end{array}$ & $\begin{array}{l}\text { Cervical cancer } \\
\text { Incidence }^{\mathrm{b}}\end{array}$ & Mortality ${ }^{\mathrm{b}}$ & $\begin{array}{l}\text { Undiscounted } \\
\text { Life-years }\end{array}$ \\
\hline \multicolumn{7}{|l|}{ Base case } \\
\hline Cytology (reflex HPV test for ASC-US) & $\$ 1,230$ & 22.856 & - & 13.31 & 4.64 & 37.978 \\
\hline HPV with genotyping (reflex cytology [ASC-US]) & $\$ 1,367$ & 22.874 & $\$ 7,667$ & 9.47 & 3.33 & 37.984 \\
\hline HPV HR only (with reflex to cytology [ASC-US]) & $\$ 1,749$ & 22.866 & Dominated & 11.14 & 3.91 & 37.981 \\
\hline Co-testing (with cytology and HPV testing) & $\$ 2,014$ & 22.868 & Dominated & 10.74 & 3.77 & 37.982 \\
\hline \multicolumn{7}{|l|}{ 1-year follow-up scenario } \\
\hline Cytology (reflex HPV test for ASC-US) & $\$ 1,230$ & 22.856 & - & 13.31 & 4.64 & 37.978 \\
\hline HPV with genotyping (reflex cytology [ASC-US]) & $\$ 1,389$ & 22.879 & $\$ 6,910$ & 8.38 & 2.95 & 37.985 \\
\hline HPV HR only (with reflex to cytology [ASC-US]) & $\$ 1,789$ & 22.879 & Dominated & 8.38 & 2.95 & 37.985 \\
\hline Co-testing (with cytology and HPV testing) & $\$ 2,059$ & 22.880 & $\$ 661,933$ & 8.17 & 2.88 & 37.986 \\
\hline
\end{tabular}

$A S C$ - $U S$ atypical squamous cells of undetermined significance, $H P V$ human papillomavirus, $H P V H R$ human papillomavirus, high-risk, ICER incremental cost-effectiveness ratio, $Q A L Y$ quality-adjusted life-year, USD United States dollars

2013 USD

b Annual rate per 100,000 women

woman screened. Co-testing generated a marginal QALY gain but was more costly than HPV with Genotyping and, by comparison, not cost effective.

In the one-way sensitivity analyses, changes in the health utilities and screening sensitivities had the largest impact on the relative cost effectiveness of HPV with Genotyping compared with the comparators (Fig. 3). HPV with Genotyping appeared more cost effective when lower utilities were assigned to the CIN and Cervical Cancer states, but remained cost effective even when the utilities were increased to 1.0. As presented in Table 5, threshold analyses showed that under the current assumptions and a WTP criterion of US\$50,000, HPV with Genotyping was less cost effective than the comparators only when (i) the current cost for HPV-16/18 genotyping was more than tripled, or (ii) the sensitivity of HPV with Genotyping was reduced by $\geq 50 \%$ of the current estimate, or (iii) the sensitivity of Cytology approached $100 \%$. Compared with Cytology, the only other non-dominated strategy, the PSA showed that, at a WTP threshold of US $\$ 50,000 / \mathrm{QALY}$ as well as with more stringent payer criteria, HPV with Genotyping was cost effective (Fig. 4).

\section{Discussion}

Stratifying cervical cancer risk via HPV-16/18 genotyping may constitute a cost-effective approach for primary screening in women aged $\geq 30$ years. Under the current assumptions, this strategy conferred incremental QALY gains and reduced cervical cancer incidence and mortality, but on average, was less expensive compared with HPV HR Only and Co-testing. HR HPV Only yielded additional re-testing and diagnostic visits and incurred higher costs in the long run compared with HPV with Genotyping. Co-testing was most costly as both routine and follow-up screening required dual testing with HPV and cytology tests. Although more costly than Cytology, HPV with Genotyping improved clinical outcomes, thereby maintaining cost effectiveness.

Because baseline sensitivities were obtained from initial screening in the ATHENA trial, the values were adjusted to account for detecting new and persistent $\geq \mathrm{CIN} 3$ at 1 year. It was expected that strategies deferring intermediate-risk women to 1-year follow-up would capture additional persistent $\geq$ CIN 3. This assumption was supported by the HPV FOCAL trial which showed that $\geq$ CIN 3 detection rates at 1 year were increased in women who had been initially high-risk HPV-positive and cytology-negative [37]. Despite identical sensitivities for $\geq$ CIN 3 in this scenario, HPV with Genotyping was cost-saving over HPV HR Only again by reducing the frequency of triage and follow-up tests. Although both strategies deferred women who were initially negative for intraepithelial lesion and malignancy to 1-year follow-up, HPV HR Only retested all high-risk HPV-positive women whereas HPV with Genotyping retested a smaller subgroup that was positive for non-16/18 high-risk HPV only. Likewise, triage was restricted to women positive for non-16/18 high-risk HPV only in the latter, but included all women positive for highrisk HPV in the former. Stratifying high-risk patients with $16 / 18$ genotyping at baseline initially incurred relatively higher routine screening and diagnostic costs, but resulted in fewer highest-risk patients in the long term, thereby reducing costs. Consistent with a prior lifetime model [11], triennial Co-testing increased QALYs in the second 
Table 4 Expected annual number of cervical screening examinations and colposcopies required for detecting $\geq$ CIN 3 in the base case and 1-year follow-up scenario

\begin{tabular}{|c|c|c|c|c|c|}
\hline \multirow[t]{2}{*}{ Strategy } & \multicolumn{4}{|c|}{ Number of screening examinations ${ }^{\mathrm{a}, \mathrm{b}}$} & \multirow{2}{*}{ No. of colposcopies per $\geq$ CIN 3 detected } \\
\hline & Routine & Triage & Retest & Colposcopies & \\
\hline \multicolumn{6}{|l|}{ Base case } \\
\hline Cytology (reflex HPV test for ASC-US) & 30,364 & 1,282 & 0 & 2,104 & 4.76 \\
\hline HPV with genotyping (reflex cytology [ASC-US]) & 23,065 & 6,372 & 1,024 & 2,159 & 3.06 \\
\hline HPV HR only (with reflex to cytology [ASC-US]) & 22,872 & 8,405 & 6,557 & 2,339 & 3.95 \\
\hline Co-testing (with cytology and HPV testing) & 22,848 & 0 & 6,686 & 2,967 & 4.79 \\
\hline \multicolumn{6}{|l|}{ 1-year follow-up scenario } \\
\hline Cytology (reflex HPV test for ASC-US) & 30,294 & 1,282 & 0 & 2,104 & 4.76 \\
\hline HPV with genotyping (reflex cytology [ASC-US]) & 23,201 & 6,265 & 1,021 & 2,130 & 2.73 \\
\hline HPV HR only (with reflex to cytology [ASC-US]) & 23,201 & 8,079 & 6,332 & 2,249 & 2.89 \\
\hline Co-testing (with cytology and HPV testing) & 23,161 & 0 & 6,479 & 2,915 & 3.67 \\
\hline
\end{tabular}

scenario, but was more costly than HPV with Genotyping, mainly due to the greater number of initial tests.

Our analysis is noteworthy as it incorporated data from over 34,000 women aged $\geq 30$ years participating in the largest US diagnostic accuracy cohort study for cervical screening to date. Contrary to other models, we employed screening performance inputs from a single trial. Screening performance can vary between clinical trials, impacted by factors such as study design, disease prevalence, and statistical methods. Applying data from the ATHENA trial facilitated comparison of the relative performance of the strategies within the same cohort, thereby reducing variability.

We note several limitations. First, excluding the impact of non-compliance at follow-up may have over-estimated screening sensitivities and costs, particularly for Co-testing and HPV HR Only, which deferred more patients to retesting. Second, we did not examine co-testing every 5 years because while this is a preferred strategy for women aged 30-65 years, the American Congress of Obstetricians and Gynecologists and other clinical experts have contended that triennial co-testing is the more likely scenario in the community as patients are unlikely to feel comfortable with extended screening intervals [10]. Thus, we believe co-testing every 3 years is relevant for a US model. Nevertheless, we recognize that screening practices may vary considerably, the effects of which may be investigated in future research.

Under the current assumptions, we projected cervical cancer incidence rates which exceeded the 2007-2011 US age-adjusted incidence of 7.8 per 100,000 reported by the SEER. However, our model included non-hysterectomized women aged $\geq 30$ years only. Rositch et al. [38] reported hysterectomy-corrected age-standardized cervical cancer incidence rates in the US. For the age groups spanning from 30 to 74 years, incidence rates (per 100,000) ranged from 11.5 to 27.4 following correction for hysterectomy prevalence [38], which was higher than our model results. These findings indicate that cervical cancer incidence may be higher than the national estimate when correcting for hysterectomy, implying the need for appropriate adjustments when evaluating cervical screening outcomes.

Annual cervical cancer mortality rates in the model exceeded the 2006-2010 SEER age-adjusted rate of 2.4 per 100,000 women. While the effects of hysterectomy prevalence on cervical cancer mortality rates have not been extensively investigated, an early study by the Centers for Disease Control and Prevention found that correcting for hysterectomy prevalence resulted in a $39 \%$ relative increase in cervical cancer mortality rates in the US during the period 1965-1988 [39]. We assumed that cancers were detected at an earlier stage in the screened population, which was associated with $90 \% 5$-year relative survival. However, this may have overestimated the projected survival benefits of screening. Various sources have reported 5 -year relative survival rates of $95 \%$ for screen-detected asymptomatic cervical cancers [30] and between 68 and $75 \%$ for patients with symptomatic presentations, although the latter would not likely represent the majority of the model population (ACS Cancer Facts and Figures, 2014; US National Institutes of Health Fact Sheet, Cervical Cancer, 2010) [30]. Further consideration of disease severity upon detection is required to more precisely predict mortality rates. 


\section{(a)}

Health utility-CIN 2,3

Cytology with reflex HPV ('Cytology') (Sensitivity)

HPV 16/18 genotyping with reflex cytology ('HPV with Genotyping') (Sensitivity) Annual discount rate (Effects) Health utility-Cervical Cancer

HPV test with genotyping (Cost) Liquid-based cytology (Cost) Office visit (routine/repeat screening) (Cost) Treatment for $\geq \mathrm{CIN} 3$ (Cost)

HPV 16/18 genotyping with reflex cytology ('HPV with Genotyping') (Specificity) Cytology with reflex HPV ('Cytology') (Specificity) Annual discount rate (Costs) Colposcopy plus biopsy (Cost) Treatment for cervical cancer (Cost) HPV DNA testing (Cost)

(b)

Health utility-CIN1 Health utility-CIN 2,3

HPV DNA test with LBC triage ('HPV HR Only') (Sensitivity) HPV 16/18 genotyping with reflex cytology ('HPV with Genotyping') (Sensitivity) HPV DNA testing (Cost) HPV test with genotyping (Cost) Office visit (routine/repeat screening) (Cost) Annual discount rate (Effects) Health utility-Cervical Cancer Annual discount rate (Costs)

HPV DNA test with LBC triage ('HPV HR Only') (Specificity) HPV 16/18 genotyping with reflex cytology ('HPV with Genotyping') (Specificity) Liquid-based cytology (Cost) Treatment for $\geq \mathrm{CIN} 3$ (Cost) Treatment for cervical cancer (Cost) Colposcopy plus biopsy (Cost)

(c)

Health utility-CIN1 Health utility-CIN 2,3

LBC and HPV DNA co-testing ('Co-testing) (Sensitivity) HPV $16 / 18$ genotyping with reflex cytology ('HPV with Genotyping') (Sensitivity) HPV DNA testing (Cost) HPV test with genotyping (Cost) Office visit (routine/repeat screening) (Cost) Annual discount rate (Costs) Liquid-based cytology (Cost)

LBC and HPV DNA co-testing ('Co-testing) (Specificity) Annual discount rate (Effects) Health utility-Cervical Cancer

HPV 16/18 genotyping with reflex cytology ('HPV with Genotyping') (Specificity) Colposcopy plus biopsy (Cost) Treatment for $\geq \mathrm{CIN} 3$ (Cost) Treatment for cervical cancer (Cost)
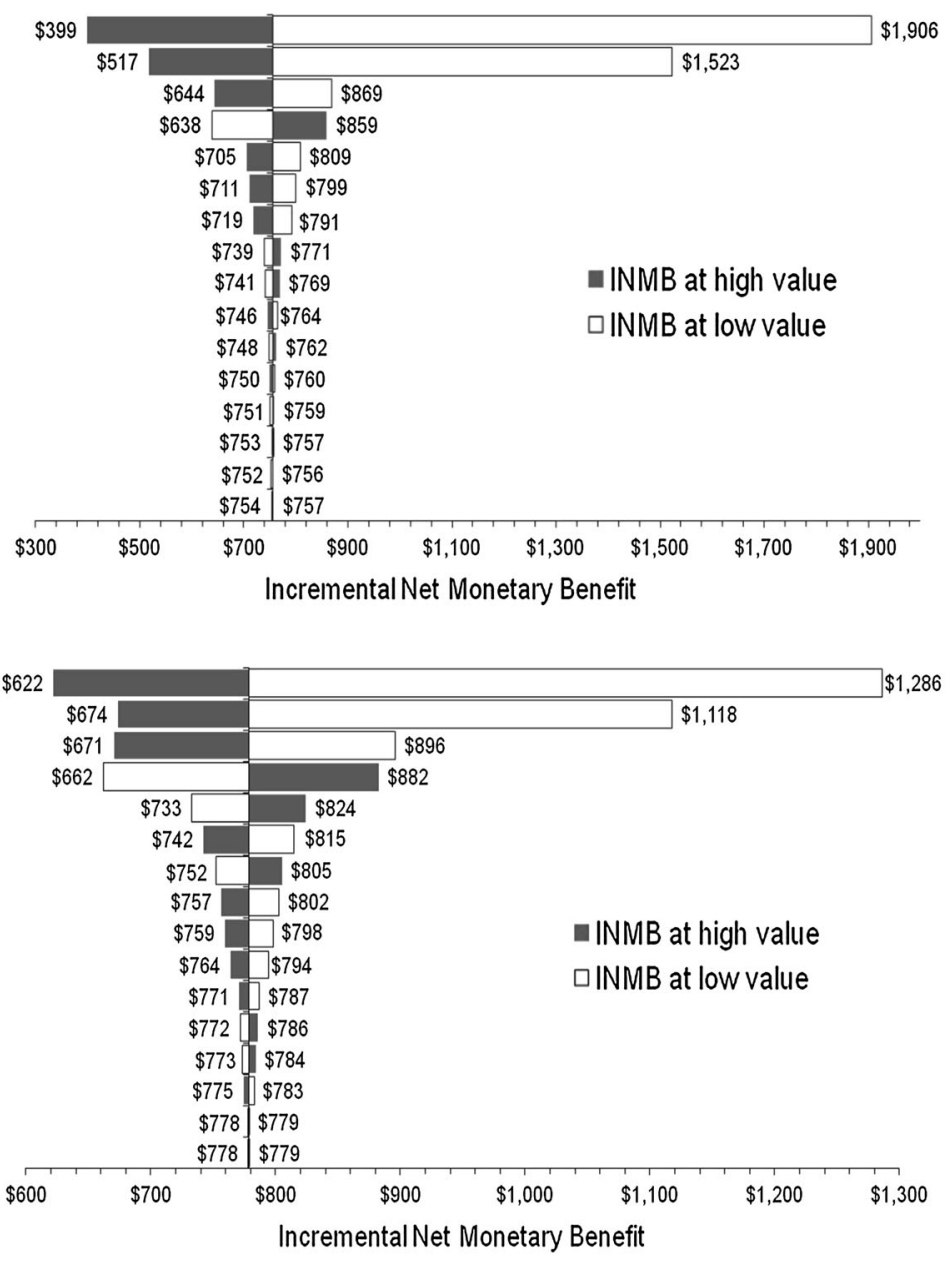

Fig. 3 One-way sensitivity analysis: tornado diagrams of INMB of HPV-16/18 genotyping with reflex cytology ('HPV with Genotyping') versus alternative primary cervical screening strategies. The INMB of HPV with Genotyping compared with Cytology (a), HPV HR Only (b), and Co-testing (c) decreased primarily when higher values were assigned to the health utilities for the CIN states, or when screening performance (sensitivity) was improved for the comparator

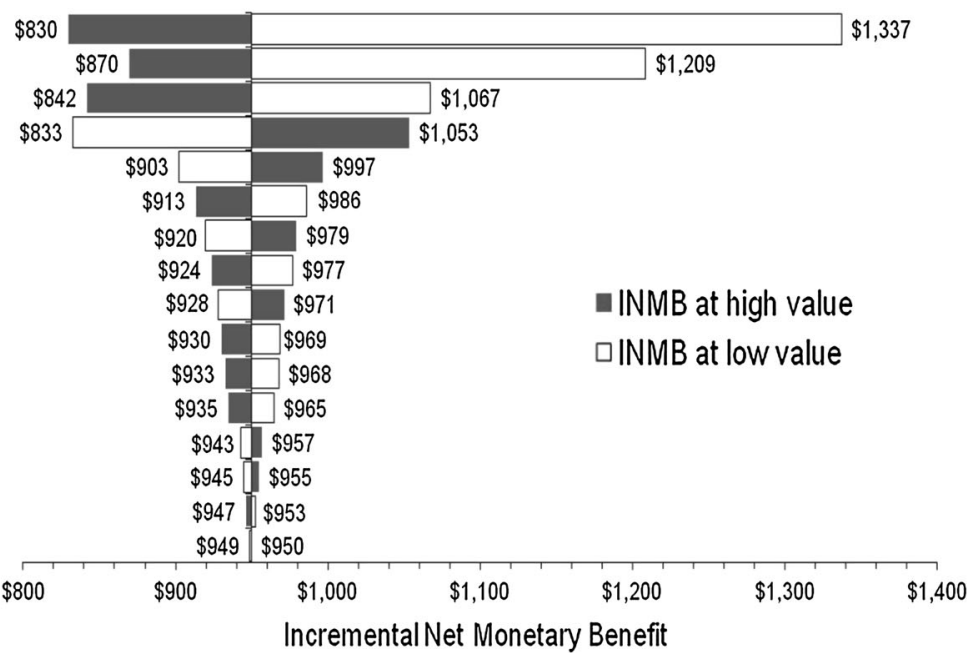

strategies or reduced for HPV with Genotyping. The INMB was calculated based on a WTP threshold of $\$ 50,000$ per QALY and represented the difference between the net monetary benefit of the two strategies. CIN cervical intraepithelial neoplasia, DNA deoxyribonucleic acid, $H P V$ human papillomavirus, $H P V H R$ human papillomavirus, high-risk, INMB incremental net monetary benefit, $Q A L Y$ quality-adjusted life year, WTP willingness-to-pay 
Table 5 Results of threshold analyses of selected model parameters in one-way sensitivity analyses

\begin{tabular}{lll}
\hline Variable & Base value & $\begin{array}{l}\text { Threshold value at which HPV with genotyping is less } \\
\text { cost effective }{ }^{\mathrm{a}} \text { than the comparator strategy }\end{array}$ \\
\hline $\begin{array}{l}\text { Cytology } \\
\text { Cytology, sensitivity }\end{array}$ & $56.1 \%$ & $>97.7 \%$ \\
HPV with genotyping, sensitivity & $72.0 \%$ & $<35.6 \%$ \\
Annual discount rate, effects & $3.0 \%$ & $>17.6 \%$ \\
cobas ${ }^{\circledR}$ HPV test with genotyping, cost & $\$ 48.24^{\mathrm{b}}$ & $>\$ 148.37^{\mathrm{b}}$ \\
HPV HR only & $51.9 \%$ & $\mathrm{HPV}$ with genotyping was cost effective at all values \\
HPV HR only, sensitivity & $72.0 \%$ & $<34.8 \%$ \\
HPV with genotyping, sensitivity & $\$ 48.24^{\mathrm{b}}$ & $>\$ 151.51^{\mathrm{b}}$ \\
cobas ${ }^{\circledR}$ HPV test with genotyping, cost & & HPV with genotyping was cost effective at all values \\
Co-testing & $56.1 \%$ & $<28.9 \%$ \\
Co-testing, sensitivity & $72.0 \%$ & $>\$ 174.18^{\mathrm{b}}$ \\
HPV with genotyping, sensitivity & $\$ 48.24^{\mathrm{b}}$ & \\
cobas ${ }^{\circledR}$ HPV test with genotyping, cost & & \\
\hline
\end{tabular}

$H P V$ human papillomavirus, $H P V H R$ human papillomavirus, high-risk, ICER incremental cost-effectiveness ratio, $Q A L Y$ quality-adjusted life year, USD United States dollars

${ }^{a}$ The ICER for HPV with genotyping exceeded $>\$ 50,000 /$ QALY (negative net monetary benefit) compared with the comparator screening strategy

b 2013 USD

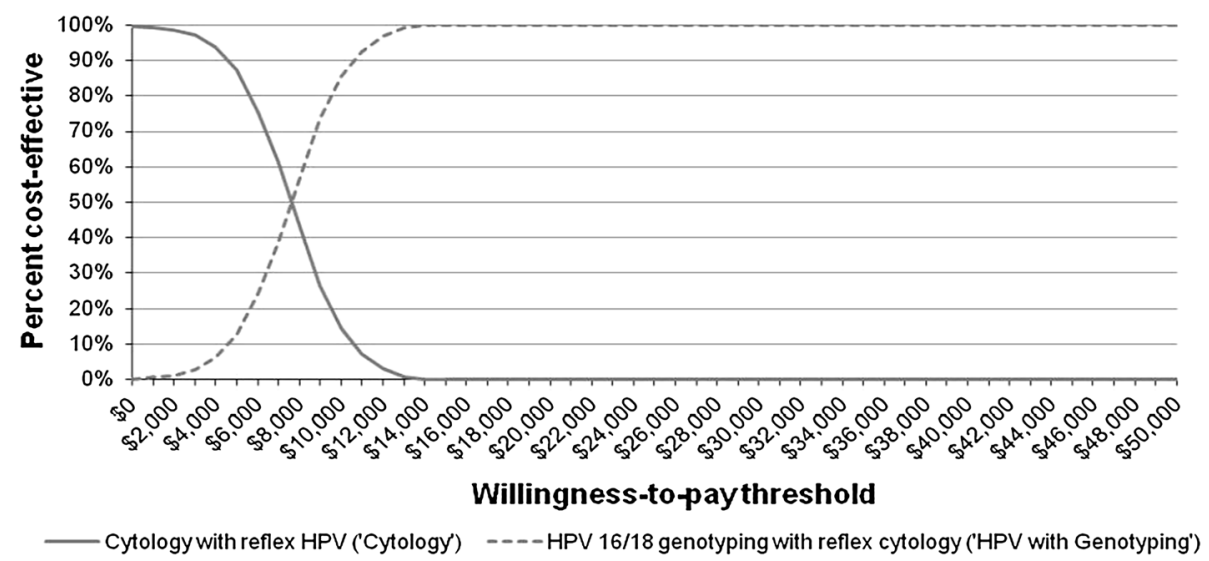

Fig. 4 Cost-effectiveness acceptability curve of the ICER for primary HPV-16/18 genotyping with reflex cytology ('HPV with Genotyping') versus cytology with reflex HPV testing ('Cytology') At a WTP threshold of $\$ 50,000$, the CEAC showed that implementing HPV with Genotyping in primary cervical screening was more likely

Lastly, we did not consider HPV immunization effects, which would theoretically decrease the prevalence of highrisk HPV and thereby reduce the sensitivity of the HPV strategies. Analyses of data from eight US managed care organizations participating in the Vaccine Safety Datalink, however, found that over the 5-year period following approval of the quadrivalent HPV vaccine (2006-2011), $56 \%$ of females ages 9-26 years had received at least one vaccine dose, but only $42 \%$ had completed the three-dose to be considered cost effective (more effective and meeting the WTP criteria) than Cytology. $C E A C$ cost-effectiveness acceptability curve, $H P V$ human papillomavirus, ICER incremental cost-effectiveness ratio, WTP willingness-to-pay

series [40]. Given these modest vaccination rates and generally lower prevalence of high-risk HPV in women $\geq 30$ years, including vaccination effects may not have substantially impacted our results.

Our findings suggest that with proper management, earlier detection of clinically relevant CIN attributable to HPV-16/18 may improve protection against cervical cancer. In the POpulation-BAsed SCreening study AMsterdam (POBASCAM), significantly more CIN $2+$ cases were 
detected at baseline in women randomized to HPV DNA and cytology co-testing compared with those tested with cytology only $(p=0.015)$ [41]. Conversely, at 5-year follow-up, significantly fewer HPV-16-positive $\geq$ CIN 3 lesions were detected with co-testing compared with cytology-based ASC-US triage $(p=0.012)$. Detection of non-HPV-16-positive $\geq$ CIN 3 did not differ between groups. These results support the utility of HPV testing in primary screening for women aged $\geq 30$ years.

Despite technological advancements, the goals of cervical cancer screening have remained unchanged and aim to identify as many women at risk for cancer as possible, while protecting others against potential harms of unnecessary interventions. Strategies employing a highly sensitive HPV test in primary screening must be balanced with a more specific test (such as cytology) to ensure that the right women receive appropriate interventions. Incorporating such a strategy, however, must account for the population screened as well as economic considerations. A Dutch simulation cost-effectiveness model evaluating nine primary screening strategies concluded that HPV screening was preferred for women aged $>30$ years in many scenarios simulating screening situations in Europe [42]. In contrast, primary cytology was preferred only in scenarios bearing high HPV prevalence and high HPV testing costs [42]. For developing countries where screening effectiveness was suboptimal, the World Health Organization recommended screening in focused geographies and in highrisk women (aged 35-49 years or 30-50 years) once or twice in their lifetime, using a highly sensitive test with high coverage of the targeted population [43].

HPV with Genotyping may detect clinically relevant high-grade CIN earlier and facilitate efficient use of healthcare resources in the long run. Detecting disease at the initial visit may also optimize protection against cervical cancer. Further analyses of women screened in real-world settings may assist in confirming the cost effectiveness of HPV-16/18 genotyping in primary cervical screening.

Acknowledgments The authors acknowledge Lionel Pinto and Trent McLaughlin, PhD for their contributions to conceptualization of the manuscript and to the review and testing of the cost-effectiveness analysis model.

Conflict of interest statements This analysis was funded by Roche Molecular Diagnostics. Dr. Huh is a non-paid consultant to Roche Molecular Diagnostics and is on the Advisory Boards for Hologic, Inc. and Becton Dickinson. J. Huang and N. Poulios are employees of Roche Molecular Diagnostics. E. Williams and T. Bramley are paid consultants to Roche Molecular Diagnostics.

Author contributions W Huh is the guarantor for the manuscript content. W. Huh, E. Williams, and J. Huang contributed to the development and design of the economic model, selection of data sources, and analyses. All authors participated in the writing, critical review, editing, and approval of the manuscript for submission.

\section{References}

1. Cuzick J, Arbyn M, Sankaranarayanan R, Tsu V, Ronco G, Mayrand $\mathrm{MH}$, et al. Overview of human papillomavirus-based and other novel options for cervical cancer screening in developed and developing countries. Vaccine. 2008;26(Suppl 10):K29-41.

2. Arbyn M, Sasieni P, Meijer CJ, Clavel C, Koliopoulos G, Dillner J. Chapter 9: Clinical applications of HPV testing: a summary of meta-analyses. Vaccine. 2006;24(Suppl 3):S3/78-89.

3. Munoz N, Bosch FX, de Sanjose S, Herrero R, Castellsague X, Shah KV, et al. Epidemiologic classification of human papillomavirus types associated with cervical cancer. $\mathrm{N}$ Engl J Med. 2003;348(6):518-27.

4. Wheeler CM, Hunt WC, Joste NE, Key CR, Quint WG, Castle PE. Human papillomavirus genotype distributions: implications for vaccination and cancer screening in the United States. J Natl Cancer Inst. 2009;101(7):475-87.

5. Khan MJ, Castle PE, Lorincz AT, Wacholder S, Sherman M, Scott DR, et al. The elevated 10-year risk of cervical precancer and cancer in women with human papillomavirus (HPV) type 16 or 18 and the possible utility of type-specific HPV testing in clinical practice. J Natl Cancer Inst. 2005;97(14):1072-9.

6. Castle PE, Stoler MH, Wright TC Jr, Sharma A, Wright TL, Behrens CM. Performance of carcinogenic human papillomavirus (HPV) testing and HPV16 or HPV18 genotyping for cervical cancer screening of women aged 25 years and older: a subanalysis of the ATHENA study. Lancet Oncol. 2011;12(9):880-90.

7. Cox JT, Castle PE, Behrens CM, Sharma A, Wright TC, Jr., Cuzick J. Comparison of cervical cancer screening strategies incorporating different combinations of cytology, HPV testing, and genotyping for HPV 16/18: results from the ATHENA HPV study. Am J Obstet Gynecol. 2012;208(3):184e1-e11.

8. Plummer M, Schiffman M, Castle PE, Maucort-Boulch D, Wheeler CM. A 2-year prospective study of human papillomavirus persistence among women with a cytological diagnosis of atypical squamous cells of undetermined significance or lowgrade squamous intraepithelial lesion. $J$ Infect Dis. 2007;195(11):1582-9.

9. Saslow D, Solomon D, Lawson HW, Killackey M, Kulasingam SL, Cain J, et al. American Cancer Society, American Society for Colposcopy and Cervical Pathology, and American Society for Clinical Pathology screening guidelines for the prevention and early detection of cervical cancer. CA Cancer J Clin. 2012;62(3):147-72.

10. Perkins RB, Anderson BL, Gorin SS, Schulkin JA. Challenges in cervical cancer prevention: a survey of U.S. obstetrician-gynecologists. Am J Prev Med. 2013;45(2):175-81.

11. Vijayaraghavan A, Efrusy MB, Goodman KA, Santas CC, Huh WK. Cost-effectiveness of using human papillomavirus 16/18 genotype triage in cervical cancer screening. Gynecol Oncol. 2010;119(2):237-42.

12. Wright TC, Jr., Stoler MH, Behrens CM, Apple R, Derion T, Wright TL. The ATHENA human papillomavirus study: design, methods, and baseline results. Am J Obstet Gynecol. 2012;206(1):46e1-e11.

13. Brismar-Wendel S, Froberg M, Hjerpe A, Andersson S, Johansson B. Age-specific prevalence of HPV genotypes in cervical cytology samples with equivocal or low-grade lesions. $\mathrm{Br} \mathbf{J}$ Cancer. 2009;101:511-7.

14. Clifford G, Franceschi S, Diaz M, et al. Chapter 3: HPV-type distribution in women with and without cervical neoplastic diseases. Vaccine. 2006;2006(24S):S3/26-S3/34.

15. Cuzick J, Cox T, Zhang G, et al. Human papillomavirus testing for triage of women with low-grade squamous lesions. Int $\mathbf{J}$ Cancer. 2013;132(4):959-66. 
16. Chesson HW, Blandford JM, Gift TL, Tao G, Irwin KL. The estimated direct medical cost of sexually transmitted diseases among American youth, 2000. Perspect Sex Reprod Health. 2004;36(1):11-9.

17. Canfell K, Barnabas R, Patnick J, Beral V. The predicted effect of changes in cervical screening practice in the UK: results from a modelling study. Br J Cancer. 2004;91(3):530-6.

18. Goldie SJ, Grima D, Kohli M, Wright TC, Weinstein M, Franco E. A comprehensive natural history model of HPV infection and cervical cancer to estimate the clinical impact of a prophylactic HPV-16/18 vaccine. Int J Cancer. 2003;106(6):896-904.

19. Sanders GD, Taira AV. Cost-effectiveness of a potential vaccine for human papillomavirus. Emerg Infect Dis. 2003;9(1):37-48.

20. Myers ER, McCrory DC, Nanda K, Bastian L, Matchar DB. Mathematical model for the natural history of human papillomavirus infection and cervical carcinogenesis. Am J Epidemiol. 2000;151(12):1158-71.

21. Insinga RP, Dasbach EJ, Elbasha EH, Liaw KL, Barr E. Progression and regression of incident cervical HPV 6, 11, 16 and 18 infections in young women. Infect Agent Cancer. 2007;2:15.

22. Hildesheim A, Schiffman MH, Gravitt PE, Glass AG, Greer CE, Zhang T, et al. Persistence of type-specific human papillomavirus infection among cytologically normal women. J Infect Dis. 1994;169(2):235-40.

23. Schiffman M, Kjaer SK. Chapter 2: Natural history of anogenital human papillomavirus infection and neoplasia. J Natl Cancer Inst Monogr. 2003;31:14-9.

24. Canfell K, Chesson H, Kulasingam SL, Berkhof J, Diaz M, Kim JJ. Modeling preventative strategies against human papillomavirus-related disease in developed countries. Vaccine. 2012;30(Suppl 5):F157-67.

25. Creighton P, Lew JB, Clements M, Smith M, Howard K, Dyer S, et al. Cervical cancer screening in Australia: modelled evaluation of the impact of changing the recommended interval from two to three years. BMC Public Health. 2010;10:734.

26. Taylor DC, Pawar V, Kruzikas D, Gilmore KE, Pandya A, Iskandar R, et al. Methods of model calibration: observations from a mathematical model of cervical cancer. Pharmacoeconomics. 2010;28(11):995-1000.

27. Gustafsson L, Ponten J, Bergstrom R, Adami HO. International incidence rates of invasive cervical cancer before cytological screening. Int J Cancer. 1997;71(2):159-65.

28. Altekruse S, Kosary C, Krapcho M, et al. SEER cancer statistics review, 1975-2007. Bethesda, MD: National Cancer Institute; 2010 (based on November 2009 data submission). Available http://seer.cancer.gov/csr/1975_2007. 2010.

29. Denny L, Wright TC. Glob. libr. women's med. Strategies for overcoming the barriers to cervical cancer screening in lowresource settings (ISSN: 1756-2228). 2009. doi:10.3843/ GLOWM.10022.

30. Andrae B, Andersson TM, Lambert PC, Kemetli L, Silfverdal L, Strander B, et al. Screening and cervical cancer cure: population based cohort study. Bmj. 2012;344:e900.
31. Massad LS, Einstein MH, Huh WK, Katki HA, Kinney WK, Schiffman M, et al. 2012 updated consensus guidelines for the management of abnormal cervical cancer screening tests and cancer precursors. J Low Genit Tract Dis. 2013;17(5 Suppl 1):S1-27.

32. Guedes AC, Zeferino LC, Syrjanen KJ, Brenna SM. Short-term outcome of cervical intraepithelial neoplasia grade 2: considerations for management strategies and reproducibility of diagnosis. Anticancer Res. 2010;30(6):2319-23.

33. Stratton KR, Durch JS, Lawrence RS, editors. Committee to Study Priorities for Vaccine Development Division of Health Promotion and Disease Prevention Institute of Medicine. Vaccines for the 21st century: a tool for decisionmaking. Washington: National Academies Press; 2000.

34. Severens JL, Milne RJ. Discounting health outcomes in economic evaluation: the ongoing debate. Value Health. 2004;7(4): 397-401.

35. Smith DH, Gravelle H. The practice of discounting in economic evaluations of healthcare interventions. Int $\mathbf{J}$ Technol Assess Health Care. 2001;17(2):236-43.

36. Briggs AH, Weinstein MC, Fenwick EA, Karnon J, Sculpher MJ, Paltiel AD. Model parameter estimation and uncertainty: a report of the ISPOR-SMDM Modeling Good Research Practices Task Force-6. Value Health. 2012;15(6):835-42.

37. Ogilvie GS, Krajden M, van Niekerk DJ, Martin RE, Ehlen TG, Ceballos K, et al. Primary cervical cancer screening with HPV testing compared with liquid-based cytology: results of round 1 of a randomised controlled trial-the HPV FOCAL Study. Br J Cancer. 2012;107(12):1917-24.

38. Rositch AF, Nowak RG, Gravitt PE. Increased age and racespecific incidence of cervical cancer after correction for hysterectomy prevalence in the United States from 2000 to 2009. Cancer. 2014;120(13):2032-8.

39. From the Centers for Disease Control. Hysterectomy prevalence, cervical cancer deaths. Jama. 1992;267(7):914.

40. Schmidt MA, Gold R, Kurosky SK, Daley MF, Irving SA, Gee J, et al. Uptake, coverage, and completion of quadrivalent human papillomavirus vaccine in the vaccine safety Datalink, July 2006June 2011. J Adolesc Health. 2013;53(5):637-41.

41. Rijkaart DC, Berkhof J, Rozendaal L, van Kemenade FJ, Bulkmans NW, Heideman DA, et al. Human papillomavirus testing for the detection of high-grade cervical intraepithelial neoplasia and cancer: final results of the POBASCAM randomised controlled trial. Lancet Oncol. 2012;13(1):78-88.

42. de Kok IM, van Rosmalen J, Dillner J, Arbyn M, Sasieni P, Iftner $\mathrm{T}$, et al. Primary screening for human papillomavirus compared with cytology screening for cervical cancer in European settings: cost effectiveness analysis based on a Dutch microsimulation model. BMJ. 2012;344:e670.

43. Sankaranarayanan R, Budukh AM, Rajkumar R. Effective screening programmes for cervical cancer in low- and middleincome developing countries. Bull World Health Organ. 2001;79(10):954-62. 